\title{
Pulmonary Dead Space Monitoring: Identifying Subjects With ARDS at Risk of Developing Right Ventricular Dysfunction
}

\author{
Alexander I Papolos, Nelson B Schiller, Annika Belzer, Hanjing Zhuo, Jeffery E Gotts, \\ Dwight Bibby, Carolyn S Calfee, and Michael A Matthay
}

\begin{abstract}
BACKGROUND: ARDS is a highly morbid condition characterized by diffuse pulmonary inflammation, which results in hypoxemic respiratory failure. Approximately $25 \%$ of patients with ARDS develop right ventricular dysfunction, with cor pulmonale being a common final pathway in a significant number of non-survivors. ARDS-related right ventricular dysfunction occurs due to acute elevation in ventricular afterload caused by mechanisms that are associated with increased pulmonary dead space fraction. Thus, we hypothesized that changes in pulmonary dead space fraction may reflect changes in pulmonary hemodynamics. METHODS: This was a prospective single-center study of 21 subjects with ARDS who underwent serial determination of pulmonary dead space fraction and pulmonary hemodynamics via transthoracic echocardiography. Linear mixed-effects modeling was performed to test for an association between a change in pulmonary dead space and a change in pulmonary hemodynamics. RESULTS: The tricuspid regurgitation velocity to right ventricular outflow track velocity time integral ratio, an echocardiographic surrogate for pulmonary vascular resistance, increased by 0.16 Wood units (Coefficient 0.16 , 95\% CI $0.09-0.23 ; P<.001)$, and the tricuspid regurgitation pressure gradient increased by $3.7 \mathrm{~mm} \mathrm{Hg}$ (Coefficient 3.7, 95\% CI $1.74-5.63, P<.001$ ) for every $10 \%$ increase in pulmonary dead space fraction. CONCLUSIONS: Increases in the pulmonary dead space fraction were associated with relative increases in both the tricuspid regurgitation velocity to right ventricular outflow track velocity time integral ratio and the tricuspid regurgitation pressure gradient, which likely contributed to the high incidence of ARDS-related right ventricular dysfunction encountered in clinical practice. Pulmonary dead space monitoring may serve as a clinical indicator to identify patients with ARDS at risk of developing right ventricular dysfunction and acute cor pulmonale. Key words: ARDS; pulmonary dead space; pulmonary vascular resistance; right ventricular failure; hypoxic pulmonary vasoconstriction. [Respir Care 2019;64(9):1101-1108. (C) 2019 Daedalus Enterprises]
\end{abstract}

\section{Introduction}

ARDS is a highly morbid condition characterized by diffuse pulmonary inflammation that results in hypoxemic respiratory failure. Approximately $25 \%$ of patients with ARDS develop right ventricular (RV) dysfunction, with

\footnotetext{
Drs Papolos, Matthay, Calfee, Schiller, Zhuo, Gotts, and Mr Bibby are affiliated with University of California San Francisco Medical Center at Parnassus, Department of Medicine.
}

Ms Belzer is affiliated with the School of Medicine at Yale University. The authors have disclosed no conflicts of interest. cor pulmonale being a common final pathway in a significant number of non-survivors. ${ }^{1}$ ARDS-related RV failure occurs due to the acute elevation in ventricular afterload caused by 3 distinct mechanisms; microvascular thrombosis related to pulmonary vascular endothelial injury, hypoxic pulmonary vasoconstriction, and vascular compression due to elevated intrathoracic pressure associ-

\footnotetext{
Correspondence: Alexander I Papolos MD, Division of Cardiology, California Medical Center, University San Francisco, 505 Parnassus Avenue, San Francisco, CA 94143. E-mail: Alexander.Papolos@ucsf.edu.
}

DOI: $10.4187 /$ respcare. 06544 
ated with mechanical ventilation (the latter being a minor contributor in the modern era of lung-protective ventilation with lower tidal volumes and reduced airway pressures)..$^{2-4}$

ARDS-related pulmonary vascular endothelial injury, microvascular thrombosis, and elevated airway pressures can contribute to an increase in pulmonary vascular resistance (PVR) and ventilation/perfusion ( $\dot{\mathrm{V} / \mathrm{Q})}$ mismatch due to decreased perfusion of ventilated alveolar units. This underperfusion of ventilated alveoli leads to an increase in physiologic pulmonary dead space (ie, the volume of each breath that participates in carbon dioxide excretion is reduced). ${ }^{5}$ In the case of focal perfusion defects, such as in the case of pulmonary embolism, elevation in pulmonary dead space and $\dot{\mathrm{V}} / \mathbf{Q}$ mismatch is partially compensated by local airway hypocapnia-induced bronchoconstriction. ${ }^{6,7}$ However, it is unknown how effective this mechanism performs in the setting of diffuse ARDS-related pulmonary microvascular thrombosis.

Pulmonary inflammation and alveolar edema disrupts alveolar gas exchange, which adds to physiologic pulmonary dead space and intrapulmonary shunting. The physiologic response to local alveolar hypoxemia by the pulmonary arterial bed is to vasoconstrict, ${ }^{8}$ which diverts deoxygenated blood to healthy alveolar units to maintain $\dot{V} / Q$ matching. ${ }^{3}$ This protective adaptation can reduce intranspulmonary shunting and systemic hypoxemia at the cost of increasing PVR. ${ }^{9}$ Thus, the predominant drivers of RV pressure overload in ARDS are associated with an increase in the pulmonary dead space fraction, which can range from $30 \%$ in normal individuals to $90 \%$ in severe ARDS. Previous studies of ARDS showed that the elevated pulmonary dead space fraction is associated with higher mortality, the development of pulmonary hypertension, and RV dysfunction. ${ }^{10-12}$ The purpose of this study was to determine if change in pulmonary dead space fraction during the early treatment course of ARDS correlates to changes in pulmonary hemodynamics detected by echocardiography.

\section{Methods}

This was a single-center, prospective, observational, cohort study of subjects with ARDS who required mechanical ventilation and were admitted to a critical care unit in the Moffitt-Long Hospital (Parnassus Campus) at the University of California, San Francisco Medical Center. Daily screening of the electronic medical record from July 1, 2017, to February 1, 2018, was used to identify subjects who met the Berlin criteria for ARDS $\left(\mathrm{P}_{\mathrm{aO}_{2}} / \mathrm{F}_{\mathrm{IO}_{2}}<300\right.$ $\mathrm{mm} \mathrm{Hg}$, PEEP $\geq 5 \mathrm{~cm} \mathrm{H}_{2} \mathrm{O}$, and bilateral infiltrates on chest radiography). ${ }^{13}$ Once identified, a suitable surrogate was contacted and informed consent for study participation was obtained. The subjects underwent serial transtho-

\section{QUICK LOOK}

\section{Current knowledge}

ARDS is associated with pulmonary vascular obstruction by mechanisms that result in elevated pulmonary dead space. Elevated pulmonary dead space is associated with mortality and right ventricular dysfunction in the setting of ARDS.

\section{What this paper contributes to our knowledge}

In the early course of ARDS, elevated pulmonary dead space was associated with relative increases in both the tricuspid regurgitation velocity to the right ventricular outflow track velocity time integral ratio and the tricuspid regurgitation pressure gradient. Pulmonary dead space monitoring may serve as a noninvasive clinical indicator to identify patients at risk of developing right ventricular dysfunction and acute cor pulmonale.

racic echocardiographic measurement of cardiac hemodynamics, with simultaneous determination of pulmonary mechanics, and pulmonary dead space fraction on days 0,2 , and 5 of enrollment. This study was approved by the institutional review board of the University of California, San Francisco. Patients were excluded if they met the Berlin criteria of ARDS for $\geq 72 \mathrm{~h}$; were $<18$ years old; had an expected survival of $<96 \mathrm{~h}$; had a previous diagnosis of pulmonary hypertension; or were receiving mechanical circulatory support of any kind, pulmonary vasodilators, or cardioplegia within $24 \mathrm{~h}$.

Baseline demographics, including age, sex, race and/or ethnicity, primary and secondary etiology of ARDS (pneumonia, aspiration, sepsis from a pulmonary source, sepsis from a non-pulmonary source, and other), comorbid conditions, and APACHE (Acute Physiology and Chronic Health Evaluation) III score were collected on enrollment. The $\mathrm{P}_{\mathrm{aO}_{2}} / \mathrm{F}_{\mathrm{IO}_{2}}$ oxygenation index, vasopressor requirement, use of pulmonary vasodilatory medications, ventilator parameters (plateau airway pressure, mean airway pressure, PEEP, tidal volume, respiratory system compliance, driving pressure), and mode of ventilation (volume control, pressure control, pressure support) were collected on enrollment and on study days 2 and 5 .

Transthoracic echocardiography was performed by using a commercially available ultrasound system (Philips Epiq, Phillips Medical Systems, Andover, Massachusetts). The recordings were subsequently analyzed by a single expert echocardiographer (NBS) who was blinded to the clinical data. RV function was determined by tricuspid annular planar systolic excursion, RV fractional area of 


\section{IDENTIFying Right VentRICULAR DySFUnCTION IN ARDS}

change, RV Doppler tissue imaging, tricuspid regurgitation pressure gradient, and RV strain imaging, as previously described by the American Society of Echocardiography. ${ }^{14}$

Tricuspid annular planar systolic excursion was measured by using the M-mode to determine the maximum systolic excursion of the lateral tricuspid annulus obtained in the apical 4-chamber view. The RV fractional area of change was defined as the percent area change between RV end-diastole and end-systole obtained in the apical 4-chamber view. RV Doppler tissue imaging was defined as peak systolic lateral tricuspid annular velocity obtained via Doppler tissue imaging from the apical 4-chamber view. The tricuspid regurgitation pressure gradient was calculated by using the modified Bernoulli equation: 4 times the peak tricuspid regurgitation velocity squared. The tricuspid regurgitation velocity was measured by continuouswave Doppler in all standard views with the assistance of intravenous agitated saline solution injection, and the highest possible value with a clean spectral Doppler envelope was used. PVR was estimated by using the Abbas regression formula ([tricuspid regurgitation velocity to RV outflow track velocity time integral $\times 10]+0.16$ ), termed the tricuspid regurgitation velocity to RV outflow track velocity time integral ratio, was obtained from the parasternal RV outflow track view. ${ }^{15} \mathrm{RV}$ strain analysis was performed on a modified apical 4-chamber view focused on optimizing the RV endocardial border definition. The RV endocardial borders were manually traced in end-systolic and end-diastolic frames to serve as the basis for semiautomatic speckle tracking to obtain RV global longitudinal strain and RV free-wall longitudinal strain (2D Cardiac Performance Analysis, TomTec, Unterschleissheim, Germany).

$\mathrm{RV}$ dysfunction was defined as having at least 2 of the 3 following echocardiographic parameters below the lower limits of normal according to the American Society of Echocardiography: tricuspid annular planar systolic excursion $<1.6 \mathrm{~cm}$, RV fractional area of change $<35 \%$, and/or RV Doppler tissue imaging $<10 \mathrm{~cm} / \mathrm{s} .{ }^{14}$ Volumetric capnography was used to calculate the partial pressure of mixed-expired $\mathrm{CO}_{2}$ (NICO Cardiopulmonary Management System, Novametrix, Wallingford, Connecticut). Standard arterial blood gas sampling was performed to determine the arterial partial pressure of $\mathrm{CO}_{2}$. Simultaneous arterial and mixed-expired $\mathrm{CO}_{2}$ determinations were applied to the Enghoff modification of the Bohr equation to determine the pulmonary dead space. ${ }^{16}$

\section{Statistical Analyses}

Continuous variables were expressed as mean $\pm \mathrm{SD}$, and categorical variables were expressed as count and percentage. The change of each variable was calculated by the difference in each variable over sequential measurements from day 0 to day 2 , and day 0 to day 5 . To evaluate the association of tricuspid regurgitation velocity to RV outflow track velocity time integral ratio with the pulmonary dead space fraction, we used linear mixed-effects modeling with a random participant effect and a random time effect, by regressing tricuspid regurgitation velocity to the $\mathrm{RV}$ outflow track velocity time integral ratio against pulmonary dead space fraction and follow-up time. The repeated time points (day 0, day 2, and day 5) were treated as a categorical variable in the model. Due to the modest sample size in this study, we used restricted maximum likelihood estimation with a Kenward-Roger correction (results reported per 10\% increase in pulmonary dead space fraction).

Similar analyses were performed to evaluate the association between pulmonary dead space fraction and tricuspid regurgitation pressure gradient, the pulmonary dead space fraction and RV function, tricuspid regurgitation velocity to $R V$ outflow track velocity time integral ratio and $\mathrm{P}_{\mathrm{aO}_{2}} / \mathrm{F}_{\mathrm{IO}_{2}}$, and the tricuspid regurgitation velocity to $\mathrm{RV}$ outflow track velocity time integral ratio and oxygenation index. The Spearman correlation coefficients were used to describe the association between the pulmonary dead space fraction and tricuspid regurgitation velocity to $\mathrm{RV}$ outflow track velocity time integral ratio at individual time points (day 0 , day 2 , and day 5 ). A 2 -sided $P$ value of $<.05$ was used to indicate statistical significance. In linear mixed-effects modeling, adjusted $P$ values by using the Bonferroni method were used for multiple comparisons. All analyses were performed by using Stata IC 15 (StataCorp, College Station, Texas).

\section{Results}

There were 21 subjects enrolled in the study. On day 0 , all 21 subjects underwent simultaneous determination of the pulmonary dead space fraction, pulmonary hemodynamics, and RV function. Due to death or liberation from mechanical ventilation, serial measurements were not possible in all the subjects. Seventeen subjects underwent testing on day 2 , and 12 subjects underwent testing on day 5 . The mean age of the subjects in the sample was 56 (range, 19-76 y) y, and $48 \%$ of the cohort were women. The etiology of ARDS was most commonly pneumonia (52\%). the severity of illness and mortality were both relatively high among the sample subjects, with mean \pm SD APACHE III scores at enrollment of $101 \pm 34$, and 30 -d survival of $29 \%$ (Table 1 ). In terms of pulmonary hemodynamics, on enrollment, the mean \pm SD tricuspid regurgitation velocity to RV outflow track velocity time integral ratio and the tricuspid regurgitation pressure gradient were above normal, at $1.93 \pm 0.48$ Wood units and $37.4 \pm 14.8 \mathrm{~mm} \mathrm{Hg}$ (Table 2), respectively. 
Table 1. Demographics

\begin{tabular}{lc}
\hline \multicolumn{1}{c}{ Variable } & \multicolumn{1}{c}{ Results } \\
\hline Age, mean \pm SD y & $56 \pm 15$ \\
Females, $(n / N) \%$ & $48(10 / 21)$ \\
Race and/or ethnicity, $(n / N) \%$ & \\
White & $48(10 / 21)$ \\
Black & $14(3 / 21)$ \\
Asian & $14(3 / 21)$ \\
Hispanic & $19(4 / 21)$ \\
Other & $5(1 / 21)$ \\
Primary and secondary etiology of ARDS & \\
Pneumonia, $(n / N) \%$ & $52(11 / 21)$ \\
Aspiration, $(n / N) \%$ & $5(1 / 21)$ \\
Sepsis from a non-pulmonary source, $(n / N) \%$ & $19(4 / 21)$ \\
Other, $(n / N) \%$ & $24(5 / 21)$ \\
APACHE III score, mean \pm SD & $101 \pm 34$ \\
30-d mortality, $(n / N) \%$ & $71(15 / 21)$
\end{tabular}

APACHE $=$ Acute Physiology and Chronic Health Evaluation

There was a significant positive correlation between the change in the pulmonary dead space fraction and the change in the tricuspid regurgitation velocity to RV outflow track velocity time integral ratio over time (Fig. 1), which increased by 0.16 Wood units for every $10 \%$ increase in pulmonary dead space fraction (Coefficient 0.16, 95\% CI 0.09-0.23; $P<.001$ ) (Table 3). Similarly, the change in the pulmonary dead space fraction was also associated with the change in tricuspid regurgitation pressure gradient (Fig. 2), which increased $3.7 \mathrm{~mm} \mathrm{Hg}$ for every $10 \%$ increase in pulmonary dead space fraction (Coefficient 3.7, 95\% CI 1.74-5.63; $P<.001$ ) (Table 3). There was no correlation between pulmonary dead space fraction and the tricuspid regurgitation velocity to RV outflow track velocity time integral ratio at individual time points; day $0(\mathrm{r}=0.03, P=.90)$, day $2(\mathrm{r}=0.06, P=.82)$, or day $5(\mathrm{r}=0.13, P=.68)$.

We also tested for an association between the change in tricuspid regurgitation velocity to $\mathrm{RV}$ outflow track velocity time integral ratio and 2 commonly used clinical indices of oxygenation, $\mathrm{P}_{\mathrm{aO}_{2}} / \mathrm{F}_{\mathrm{IO}_{2}}$, and the oxygenation index. However, no association between the change in tricuspid regurgitation velocity to $\mathrm{RV}$ outflow track velocity time integral ratio and either the change in $\mathrm{P}_{\mathrm{aO}_{2}} / \mathrm{F}_{\mathrm{IO}_{2}}$ (Coefficient $-0.0002,95 \% \mathrm{CI}-0.002$ to $0.002 ; P=.82)$, or the change in oxygenation index (Coefficient 0.004, $95 \%$ CI -0.012 to $0.020 ; P=.59$ ) was observed.

RV dysfunction developed in 5 subjects (24\%), each of whom had a mean \pm SD tricuspid regurgitation velocity to $\mathrm{RV}$ outflow track velocity time integral ratio above the upper limits of normal ( $2 \pm 0.34$ Wood units). There was a weak correlation between the change in the pulmonary dead space fraction and the change in both tricuspid an- nular planar systolic excursion (Coefficient $0.10,95 \% \mathrm{CI}$ $0.002-0.21 ; P=.047)$, and RV free-wall longitudinal strain (Coefficient $-1.59,95 \%$ CI -3.05 to -0.12 ; $P=.034$ ) (Table 3 ). This association was not statistically significant after Bonferroni adjustment. There was no association between the change in pulmonary dead space fraction and the change in the RV fractional area of change (Coefficient $0.74,95 \% \mathrm{CI}-0.89$ to $2.36 ; P=.38$ ), $\mathrm{RV}$ Doppler tissue imaging (Coefficient 0.23 , 95\% CI -0.57 to $1.03 ; P=.57$ ), or RV global longitudinal (Coefficient $0.71,95 \% \mathrm{CI}-1.51$ to $0.08 ; P=.08)$.

\section{Discussion}

The main finding of this study was that the change in pulmonary dead space fraction was associated with the change in tricuspid regurgitation velocity to $\mathrm{RV}$ outflow track velocity time integral ratio and tricuspid regurgitation pressure gradient during the early course of ARDS. Specifically, the tricuspid regurgitation velocity to RV outflow track velocity time integral ratio increased by 0.16 Wood units and tricuspid regurgitation pressure gradient increased by $3.7 \mathrm{~mm} \mathrm{Hg}$ for every $10 \%$ increase in the pulmonary dead space fraction.

The incidence of ARDS in the United States approximates 200,000 cases annually. ${ }^{17}$ Although widespread adoption of lung protective ventilation has improved outcomes, ARDS remains a highly morbid condition associated with a $39 \%$ mortality. ${ }^{17}$ A series of studies reported that roughly $25 \%$ of subjects with ARDS develop RV dysfunction and/or cor pulmonale.1,10,18-20 Those in whom invasive pulmonary hemodynamic monitoring was available found that an elevated mean pulmonary arterial systolic pressure and/or PVR was associated with significantly higher mortality. ${ }^{10,19,20}$

We did not detect a correlation between the change in tricuspid regurgitation velocity to $\mathrm{RV}$ outflow track velocity time integral ratio and the change in the measurements of oxygenation commonly used in the clinical treatment of ARDS, including the $\mathrm{P}_{\mathrm{aO}_{2}} / \mathrm{F}_{\mathrm{IO}_{2}}$ (which defines ARDS severity by the Berlin definition and serves as enrollment criteria for clinical trials), ${ }^{13}$ and the oxygenation index (which may be superior to the $\mathrm{P}_{\mathrm{aO}_{2}} / \mathrm{F}_{\mathrm{IO}_{2}}$ for predicting ARDS-related mortality). ${ }^{21}$ These measures of oxygenation quantify the degree of intrapulmonary shunting that occurs through perfused but poorly ventilated alveoli. In contrast, the pulmonary dead space fraction reflects both intrapulmonary shunting and the degree to which ventilated alveoli are being poorly perfused and, hence, the severity of increased RV afterload caused by pulmonary microvascular thrombosis, hypoxic pulmonary vasoconstriction, and pneumatic vascular compression. 
Table 2. Subject Characteristics

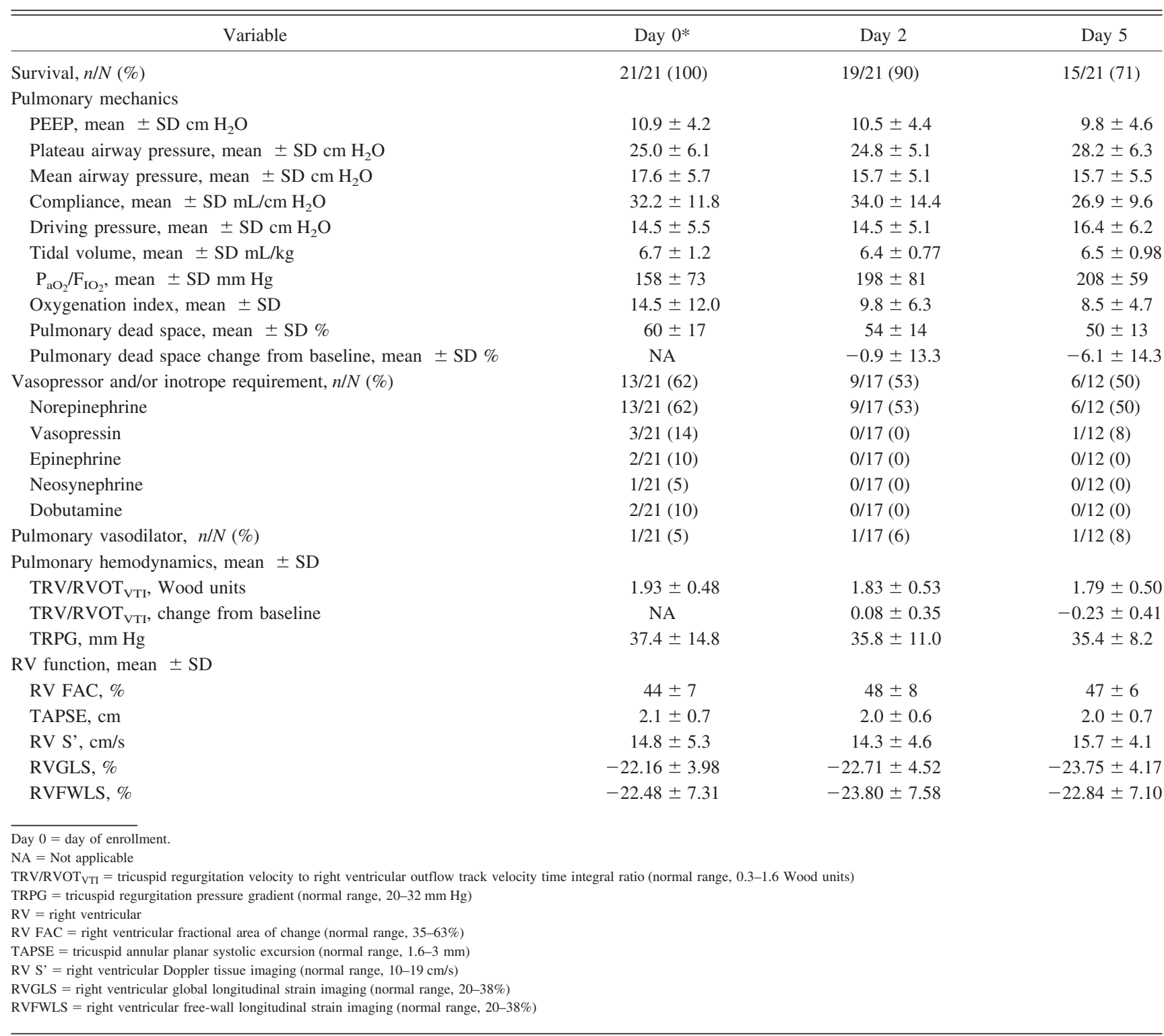

The right ventricle is a thin-walled structure that pumps blood through a low-pressure, high-capacitance vascular bed. Its compliance allows it to tolerate large shifts in preload while making it ill equipped to overcome acute increases in afterload. ${ }^{22}$ Pulmonary vascular obstruction associated with ARDS is a relatively acute process that does not allow for compensatory RV remodeling, which is why RV failure is so commonly encountered. There is ongoing interest in the clinical importance of ARDS-related pulmonary hypertension and RV dysfunction, in part because the current data are conflicting on its association with mortality. ${ }^{1,18}$ Regardless, knowledge of the presence of RV dysfunction is clinically meaningful, and, thus, we interpreted the findings of this study to support the use of pulmonary dead space monitoring as a clinical indicator that identifies patients at risk of developing RV dysfunction. Measurement of the pulmonary dead space fraction is noninvasive and is a standard practice in some ICUs in patients with ARDS who are mechanically ventilated. ${ }^{23}$ As such, we believe the relationship described by this study was important information for critical care clinicians. In addition, although none of the subjects in our sample had preexisting pulmonary arterial hypertension, we speculated that such patients are likely at higher risk of developing ARDS-related RV dysfunction, given that they have less pulmonary vascular reserve.

Similar to other large studies of ARDS, $\sim 25 \%$ of our subjects in the sample developed RV dysfunction. ${ }^{1}$ We did 


\section{IDENTIFYING Right VENTRICULAR Dysfunction IN ARDS}

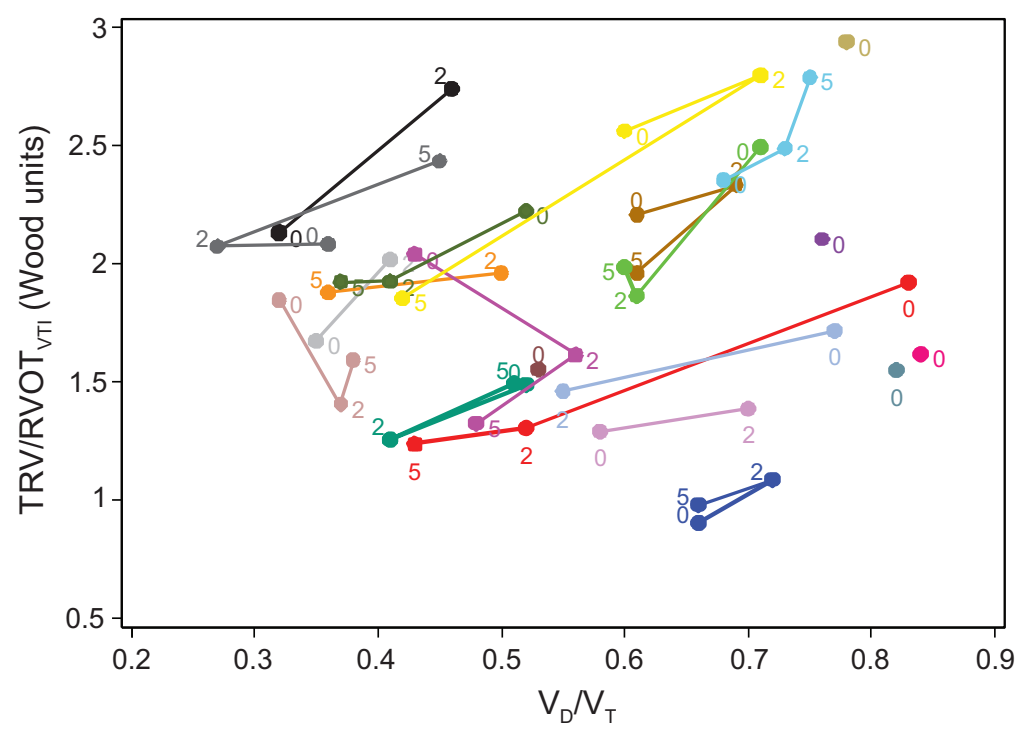

Fig. 1. Scatter plot, showing the subject-specific relationship between pulmonary dead space fraction and the tricuspid regurgitation velocity to right ventricular outflow track velocity time integral ratio (TRV/RVOT $\left.\mathrm{VTI}_{1}\right)$. On days $0(n=21), 2(n=17)$, and 5 ( $\left.n=12\right)$. Information of each subject is color-coded; serial measurements are connected by a line. There are 6 subjects in whom only a single measurement of dead space and TRV/RVOT ${ }_{V T I}$ was possible because the subject died or was extubated.

Table 3. Linear Mixed-Effects Model Analysis of Echocardiographic Variables in Relation to Change in Pulmonary Dead Space Fraction

\begin{tabular}{lccc}
\hline \hline \multicolumn{1}{c}{ Variable } & Coefficient & $95 \%$ CI & $P^{*}$ \\
\hline TRV/RVOT $_{\text {VTI }}$, Wood units & 0.16 & $0.09-0.23$ & $<.001$ \\
TRPG, mm Hg & 3.68 & $1.74-5.63$ & $<.001$ \\
RV FAC, \% & 0.74 & -0.89 to 2.36 & .38 \\
TAPSE, cm & 0.10 & $0.002-0.21$ & .047 \\
RV S', cm/s & 0.23 & -0.57 to 1.03 & .57 \\
RVGLS, \% & -0.71 & -1.51 to 0.08 & .08 \\
RVFWLS, \% & -1.59 & -3.05 to -0.12 & .034
\end{tabular}

Results are reported per $10 \%$ increase in pulmonary dead space fraction.

$P$ value $<.0056$ for statistical significance after Bonferroni adjustment.

$\mathrm{TRV}_{\mathrm{RVOT}} \mathrm{T}_{\mathrm{VTI}}=$ tricuspid regurgitation velocity to right ventricular outflow track velocity

time integral ratio (normal range, 0.3-1.6 Wood units)

$\mathrm{TRPG}=$ tricuspid regurgitation pressure gradient (normal range, $20-32 \mathrm{~mm} \mathrm{Hg}$ )

$\mathrm{RV} F A C=$ right ventricular fractional area of change (normal range, 35-63\%)

TAPSE $=$ tricuspid annular planar systolic excursion (normal range, $1.6-3 \mathrm{~mm}$ )

RV S' = right ventricular Doppler tissue imaging (normal range, $10-19 \mathrm{~cm} / \mathrm{s}$ )

RVGLS $=$ right ventricular global longitudinal strain imaging (normal range, -20 to $-38 \%$ )

RVFWLS $=$ right ventricular free-wall longitudinal strain imaging (normal range, -20

to $-38 \%$ )

not detect an association between the change in pulmonary dead space fraction and the change in RV fractional area of change, tricuspid annular planar systolic excursion, RV Doppler tissue imaging, RV global longitudinal, or RV free-wall longitudinal strain. We recognized that the study was not powered for this end point, and assessment of intrinsic RV function was confounded by the provisional administration and titration of clinically indicated inotropic agents in two thirds of the population.
The main limitations of this study were the modest sample size and the inability to perform invasive measurement of pulmonary hemodynamics. However, the Abbas formula used to estimated PVR ([tricuspid regurgitation velocity to RV outflow track velocity time integral ratio $32 \times 10]+0.16$ ) has previously been validated and shown to have a strong correlation with the invasive measurement of PVR (r 0.929, 95\% CI 0.8-0.96). ${ }^{15}$ Due to low utilization of central venous catheters in the sample, we were unable to transduce right atrial pressure and, as such, the tricuspid regurgitation pressure gradient and not the pulmonary arterial systolic pressure was reported. Given the observational nature of the study, we were unable to control for confounding factors that affect the determination of $\mathrm{RV}$ function or the pulmonary dead space fraction, such as ventricular loading conditions, ventriculo-ventricular interactions, intrathoracic interactions, arrhythmias, cardiotoxic and/or cardiotropic medications, and vasoactive and/or bronchoactive medication. We attempted to address these confounding issues by carrying out our analyses on changes observed within each subject over time so that each patient served as his or her own control.

\section{Conclusions}

Increases in the pulmonary dead space fraction were associated with relative increases in both the tricuspid regurgitation velocity to $\mathrm{RV}$ outflow track velocity time integral ratio and the tricuspid regurgitation pressure gradient, which likely contributes to the high incidence of ARDS-related RV 


\section{IDENTIFYING Right VeNTRICULAR DYSFUNCTION IN ARDS}

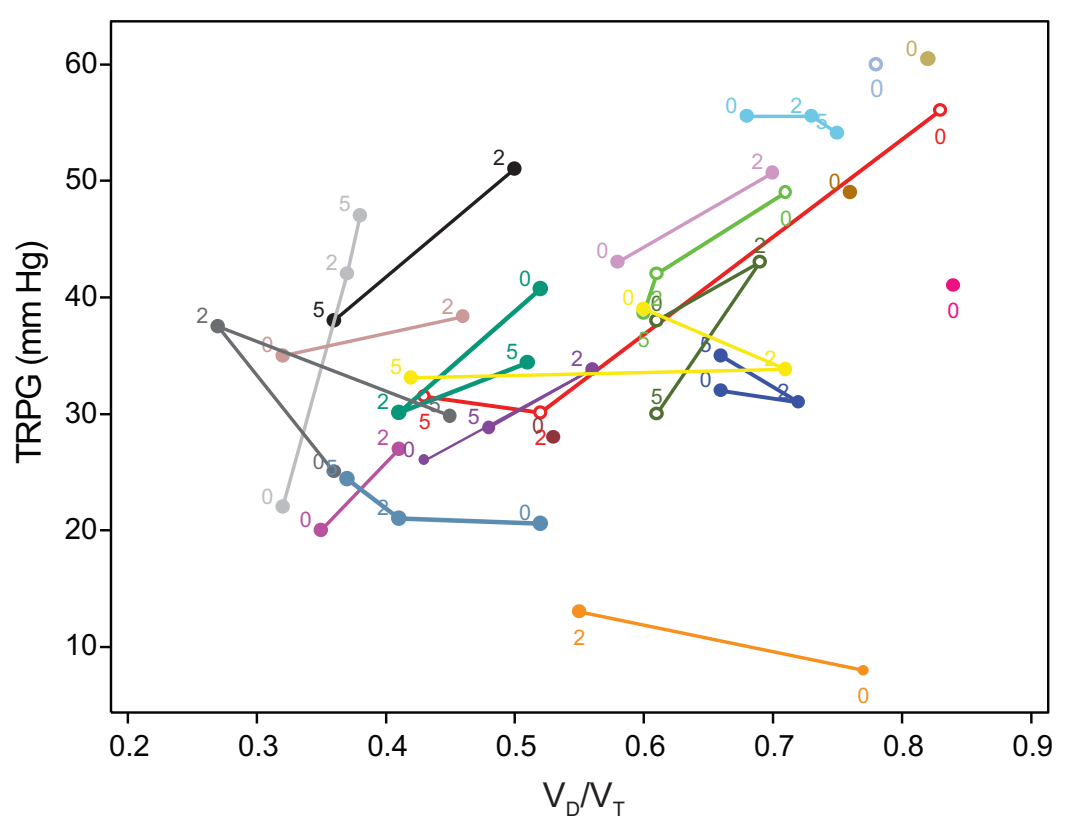

Fig. 2. Scatter plot, showing the subject-specific relationship between pulmonary dead space fraction and the tricuspid regurgitation pressure gradient (TRPG) on days $0(n=21), 2(n=17)$, and $5(n=12)$. Information of each subject is color-coded; serial measurements are connected by a line. There were 6 subjects in whom only a single measurement of dead space and TRPG was possible because the subjects died or were extubated.

dysfunction encountered in clinical practice. Pulmonary dead space monitoring may serve as a clinical indicator to identify patients with ARDS at risk of developing RV dysfunction and acute cor pulmonale.

\section{ACKNOWLEDGMENTS}

This research was supported by a grant from Bayer Pharmaceuticals.

\section{REFERENCES}

1. Mekontso Dessap A, Boissier F, Charron C, Bégot E, Repessé X, Legras A, et al. Acute cor pulmonale during protective ventilation for acute respiratory distress syndrome: prevalence, predictors, and clinical impact. Intensive Care Med 2016;42(5):862-870.

2. Tomashefski JF Jr, Davies P, Boggis C, Greene R, Zapol WM, Reid LM. The pulmonary vascular lesions of the adult respiratory distress syndrome. Am J Pathol 1983;112(1):112-126.

3. Lahm T, McCaslin CA, Wozniak TC, Ghumman W, Fadl YY, Obeidat OS, et al. Medical and surgical treatment of acute right ventricular failure. J Am Coll Cardiol 2010;56(18):1435-1446.

4. Schmitt JM, Vieillard-Baron A, Augarde R, Prin S, Page B, Jardin F. Positive end-expiratory pressure titration in acute respiratory distress syndrome patients: impact on right ventricular outflow impedance evaluated by pulmonary artery Doppler flow velocity measurements. Crit Care Med 2001;29(6):1154-1158.

5. Robertson HT. Dead space: the physiology of wasted ventilation. Eur Respir J 2015;45:1704-1716.

6. Severinghaus JW, Swenson EW, Finley TN, Lategola MT, Williams J. Unilateral hypoventilation produced in dogs by occluding one pulmonary artery. J Appl Physiol 1961;16:53-60.

7. Swenson EW, Finley TN, Guzman SV. Unilateral hypoventilation in man during temporary occlusion of one pulmonary artery. J Clin Invest 1961;40(5):828-835.
8. Naeije R, Dedobbeleer C. Pulmonary hypertension and the right ventricle in hypoxia. Exp Physiol 2013;98(8):1247-1256.

9. Sylvester JT, Shimoda LA, Aaronson PI, Ward JP. Hypoxic pulmonary vasoconstriction. Physiol Rev 2012;92(1):367-520.

10. Bull TM, Clark B, McFann K, Moss M, National Institutes of Health/ National Heart, Lung, and Blood Institute ARDS Network. Pulmonary vascular dysfunction is associated with poor outcomes in patients with acute lung injury. Am J Respir Crit Care Med 2010; 182(9):1123-1128.

11. Kallet RH, Zhuo H, Liu KD, Calfee CS, Matthay MA, National Heart Lung and Blood Institute ARDS Network Investigators. The association between physiologic dead-space fraction and mortality in subjects with ARDS enrolled in a prospective multi-center clinical trial. Respir Care 2014;59(11):1611-1618.

12. Nuckton TJ, Alonso JA, Kallet RH, Daniel BM, Pittet JF, Eisner MD, Matthay MA. Pulmonary dead-space fraction as a risk factor for death in the acute respiratory distress syndrome. $\mathrm{N}$ Engl $\mathrm{J}$ Med 2002;346(17):1281-1286.

13. ARDS Definition Task Force, Ranieri VM, Rubenfeld GD, Thompson BT, Ferguson ND, Caldwell E, et al. Acute respiratory distress syndrome: the Berlin Definition. JAMA 2012;307(23):2526-2533.

14. Rudski LG, Lai WW, Afilalo J, Hua L, Handschumacher MD, Chandrasekaran K, et al. Guidelines for the echocardiographic assessment of the right heart in adults: a report from the American Society of Echocardiography endorsed by the European Association of Echocardiography, a registered branch of the European Society of Cardiology, and the Canadian Society of Echocardiography. J Am Soc Echocardiogr 2010; 23(7):685-713; quiz 786-688.

15. Abbas AE, Fortuin FD, Schiller NB, Appleton CP, Moreno CA, Lester SJ. A simple method for noninvasive estimation of pulmonary vascular resistance. J Am Coll Cardiol 2003;41(6):1021-1027.

16. Cepkova M, Kapur V, Ren X, Quinn T, Zhuo H, Foster E, et al. Pulmonary dead space fraction and pulmonary artery systolic pres- 


\section{IDENTIFying Right VentRICULAR DySFUnCTION IN ARDS}

sure as early predictors of clinical outcome in acute lung injury. Chest 2007;132(3):836-842.

17. Rubenfeld GD, Caldwell E, Peabody E, Weaver J, Martin DP, Neff $\mathrm{M}$, et al. Incidence and outcomes of acute lung injury. N Engl J Med 2005;353(16): 1685-1693.

18. Boissier F, Katsahian S, Razazi K, Thille AW, Roche-Campo F, Leon $\mathrm{R}$, et al. Prevalence and prognosis of cor pulmonale during protective ventilation for acute respiratory distress syndrome. Intensive Care Med 2013;39(10):1725-1733.

19. Osman D, Monnet X, Castelain V, Anguel N, Warszawski J, Teboul JL, et al.; French Pulmonary Artery Catheter Study Group. Incidence and prognostic value of right ventricular failure in acute respiratory distress syndrome. Intensive Care Med 2009;35(1):69-76.
20. Squara P, Dhainaut JF, Artigas A, Carlet J. Hemodynamic profile in severe ARDS: results of the European Collaborative ARDS Study. Intensive Care Med 1998;24(10):1018-1028.

21. Dechert RE, Park PK, Bartlett RH. Evaluation of the oxygenation index in adult respiratory failure. J Trauma Acute Care Surg 2014; 76(2):469-473.

22. Ventetuolo CE, Klinger JR. Management of acute right ventricular failure in the intensive care unit. Ann Am Thorac Soc 2014;11(5): 811-822.

23. Kallet RH, Zhuo H, Ho K, Lipnick MS, Gomez A, Matthay MA. Lung injury etiology and other factors influencing the relationship between dead-space fraction and mortality in ARDS. Respir Care 2017;62(10):1241-1248 